\title{
Optimal 4- and 8-State Across-the-subchannels TCM Encoders for DMT Systems
}

\author{
V. Shashidhar \\ Department of Electrical \\ Communication Engineering \\ Indian Institute of Science \\ Bangalore 560012 \\ India \\ shashidhareprotocol.ece.iisc.ernet.in
}

\author{
B. Sundar Rajan ${ }^{1}$ \\ Department of Electrical \\ Communication Engineering \\ Indian Institute of Science \\ Bangalore 560012 \\ India \\ bsrajaneece.iisc.ernet.in
}

\author{
V .Umapathi Reddy ${ }^{2}$ \\ Department of Electrical \\ Communication Engineering \\ Indian Institute of Science \\ Bangalore 560012 \\ India \\ vurece.iisc.ernet.in
}

Abstract - We give the optimal 4- and 8-state trellises for across-the-subchannels TCM for DMT systems.

\section{INTRODUCTION}

TCM can be performed for DMT systems in two ways : coding parallely and coding across the subchannels. The decoding delay in the latter case is $M$ times less than that in the former case, where $M$ is the number of subchannels [1]. We refer the latter as across-the-subchannels TCM for DMT systems.

At the receiever input, the SNR's in different subchannels are different due to the channel impulse response. Thus, the minimum weighted Euclidean distance becomes the decision criteria for ML decoding, and hence we use weighted Viterbi decoding. Due to this weighting, the best trellis known for single carrier systems need not be the best in our case.

\section{Classification of Trellises}

We classify all the $S$-state trellises into $\gamma$ classes (where $\gamma$ $\left.=\log _{2} S\right)$ as $\left\{S^{\left(2^{x} ; p\right)}: 1 \leq x \leq \gamma\right\}$, where $S^{\left(2^{x} ; p\right)}$ denotes an $S$-state trellis with a node at a level connected to $2^{x}$ nodes in the next level and having $2^{p}$ parallel transitions. We label the top most node as $s_{0}$ and the last node as $s_{2} \gamma_{-1}$.

Definition 1: A cyclic trellis is a trellis in which the branches diverging from a node $s_{n}$ at any level connect to $2^{b-p}$ nodes

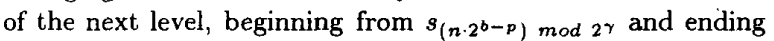

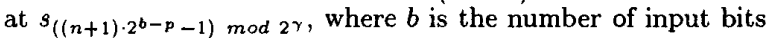
per symbol.

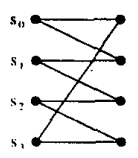

(a)

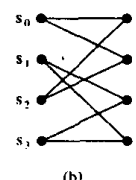

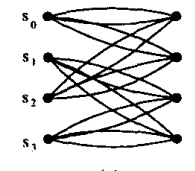

(c)

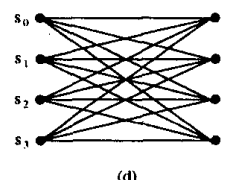

Figure 1: Some possible 4-state trellises : (a) $4^{(2 ; 0)}$ non-cyclic (b) $4^{(2 ; 0)}$ cyclic (c) $4^{(2 ; 1)}$ cyclic (d) $4^{(4 ; 0)}$ cyclic

Definition 2: The Convergence length of a trellis is defined as the minimum of all lengths of pairs of paths that diverge from a node, excepting the parallel transitions, and converge at another node.

${ }^{1}$ This work was partly supported by CSIR, India, through Research Grants (No:25(0086)/97/EMRI-II) and (22(0298)/99/EMRII) to B.S.Rajan

${ }^{2}$ This work was supported in part by DARPA Grant F49620-951-0525-P00005 during his stay at Stanford university.
The upper bound on the convergence length of a trellis is given by $[2]$

$$
L_{\max }=\left\lfloor\frac{\gamma}{b_{1}}\right\rfloor+1
$$

where $b_{1}$ refers to that part of the input bits which affects the state of encoder and $\lfloor x\rfloor$ denotes the largest integer less than or equal to $x$.

Theorem 1: The convergence length of a cyclic trellis is equal to $L_{\max }$, i.e., cyclic trellises achieve the upper bound on the convergence length.

\section{Optimal 4- and 8-State Trellises}

Let $b_{\min }=\min _{i \in\{0, M-1]}\left\{b_{i}\right\}$, where $b_{i}$ is the number of input bits in $i^{\text {th }}$ subchannel and $s_{t} w_{t}=\min _{i \in[0, M-1]}\left\{s_{i} w_{i}\right\}$, where $s_{i}$ and $w_{i}$ are the squared miniumum Euclidean distance of the $i^{\text {th }}$ subchannel symbol constellation and weighting factor for that subchannel, respectively.

Theorem 2 : The best trellis for 4-state across-thesubchannels TCM is

(a) the $4^{(2 ; 0)}$ cyclic trellis, for $b_{\min }=1$,

(b) the $4^{\left(4 ; b_{m i n}-2\right)}$ cyclic trellis if

$$
\min _{i \in\{0, M-1]}\left\{2 s_{i} w_{i}+s_{i \oplus 1} w_{i \oplus 1}\right\}>4 s_{t} w_{t}
$$

else the $4^{\left(2 ; b_{\min }-1\right)}$ cyclic trellis, for $b_{\min } \geq 2$.

Theorem 3 : The best trellis for 8-state across-thesubchannels TCM is

(a) the $8^{(2 ; 0)}$ cyclic trellis, for $b_{\min }=1$,

(b) the $8^{(4 ; 0)}$ cyclic trellis, for $b_{\min }=2$,

(c) the $8^{\left(8 ; b_{\min }-3\right)}$ cyclic trellis if

$$
\min _{i, k \in[0, M-1]}\left\{2 s_{i} w_{i}+s_{k} w_{k}\right\}>8 s_{t} w_{t}
$$

else the $8^{\left(4 ; b_{\min }-2\right)}$ cyclic trellis, for $b_{\min } \geq 3$.

\section{ACKNOWLEDGMENTS}

B.S.Rajan gratefully acknowledges IBM India Research Lab, for the travel support to present this paper.

\section{REFERENCES}

[1] J.A. C. Bingham, "Multicarrier Modulation for Data Transmission: An Idea Whose Time Has Come," IEEE Communication Magazine, pp.5-14, May 1990.

[2] S. Hamidreza Jamali and Tho Le-Ngoc, "Coded-modulation Techniques for Fading Channels," Kluwer Academic Publishers, 1994. 